\title{
The influence of nationality and playing position on relative age effects in rugby union: A cross-cultural comparison
}

\author{
P E Kearney, $\mathrm{PhD}$ \\ ${ }^{1}$ Physical Education \& Sport Sciences Department, University of Limerick, \\ Limerick, Ireland
}

\section{Corresponding author: P E Kearney (kearneype@gmail.com)}

Background: Recent research in a French context suggested that relative age effects (RAEs) in rugby union may be influenced by playing position; specifically, that RAEs may be more pronounced in back row players who do not have as extreme an anthropomorphic profile as other forward positions.

Methods: In the present study, dates of birth of 6663 players from four nations (Australia, England, New Zealand, South Africa) were analysed for relative age effects.

Results: The hypothesis that RAEs would be more pronounced in back row players was not supported. South African rugby was an obvious outlier due to the finding that RAEs were present across all playing units. These results suggest that late maturing players have been disproportionately lost to the South African system across all positions.

Conclusion: Nation-specific youth sport culture appears to be more important than playing position for determining who is at risk of RAEs in rugby union.

Keywords: birth date, team sport, talent development

\section{S Afr J Sports Med 2017;29:1-4. DOI: 10.17159/2078-516X/2017/v29i0a1886}

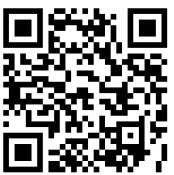

In order to provide appropriate competition, children participating in youth sport are typically assigned to cohorts based on their age in relation to a specific cut-off date (e.g. 1 January). Although birth rates tend to remain uniform across the year, examination of representative youth and professional sports teams often reveals a higher number of individuals who were born in the months immediately following the cut-off date. ${ }^{[1]}$ The most common explanation for this phenomenon, termed the relative age effect (RAE), is that children born in the months immediately after the cut-off date are, on average, more advanced in their physical development. [2] This initial advantage is thought to be compounded as coaches may confuse this developmental advantage for a difference in potential and provide additional opportunities to relatively early maturing youths in the form of supplementary coaching or access to higher levels of competition. $[1,3]$

Rugby union is a contact sport, in which players attempt to carry a ball over their opponents' touch-in-goal line while evading tacklers from the opposing team. Consequently, physical size, strength and speed are critical both in attack and defence. As a result, rugby union is a strong candidate for RAEs. ${ }^{[1,3]}$ Indeed, World Rugby ${ }^{[4]}$ and National Governing Bodies $^{[5]}$ have provided guidance to coaches and administrators relating to RAEs and/or the variation in player maturation that these effects represent. Furthermore, a number of countries have introduced modified games or weight categories in some categories of youth rugby in an attempt to negate the effect of variations in player maturation on participation and performance. ${ }^{[5]}$

It was recently suggested that the existence of RAEs within rugby union may depend upon playing position ${ }^{[6]}$, due to the diversity of body morphologies present within the game. ${ }^{[7]}$ Certain positions (front row, second row) might be protected from RAEs due to these positions requiring specific body shapes. ${ }^{[6]}$ For example, second row forwards are typically the tallest players on the pitch due to their role of competing for the ball in the air, while front row forwards are typically the heaviest, due to their role emphasising direct physical competition with opponents for possession. [7] Consequently, a player who shows the potential to grow to a large stature may be retained in a squad despite them not being as physically mature as his/her peers. Consistent with that hypothesis, an examination of French professional rugby union players demonstrated a RAE for back row forwards, but not for front row or second row forwards. ${ }^{[6]}$ No RAE was found for French backline players. The aim of this study was to examine whether the same finding held in other countries.

\section{Methods}

A review of an online database (http://www.itsrugby.co.uk/) revealed the names of 8751 senior male professional rugby players of Australian, English, New Zealand or South African nationality. Removing players for whom no date of birth was available, or who were born before 1975, produced the eventual sample of 6663 players. Players were grouped according to the main positional division in rugby union into forwards or backs, as well as to playing units ${ }^{[7]}$ (see Figure 1).

Consistent with the selection year (starting in January in Australia, New Zealand and South Africa; September in England), players' birth dates were categorised into four quarters. Chi-squared goodness of fit tests were used to examine whether the distribution of births differed from that of an even distribution. Cohen's w provided a measure of effect size, with $\mathrm{w}$ values of $0.1,0.3$ and 0.5 indicating a small, medium and large effect size, respectively. Where significant chi-square results were found, standardised residuals (SR) provided a post-hoc test to identify in which quarters there were significant deviations from the expected frequencies. A positive SR indicated a higher than expected number of births in that quarter. A negative SR indicated a lower than expected number of births in that quarter. SRs $\geq \pm 1.96$ were deemed noteworthy.

\section{Results}

Overall results are presented in Table 1 . When considering all players, RAEs were evident in all four samples. With regard to specific positions (Table 2 and Figure 1), the hypothesis that RAEs would be more prevalent in back row players than in 
Table 1. Relative age distribution of rugby union players according to nationality and playing unit

\begin{tabular}{|c|c|c|c|c|c|c|c|c|c|c|c|c|}
\hline Country & Population & $\mathbf{N}$ & $\%$ Q1 & $\%$ Q2 & $\%$ Q3 & $\%$ Q4 & $\mathbf{P}$ & $\mathbf{w}$ & SR Q1 & SR Q2 & SR Q3 & SR Q4 \\
\hline \multirow[t]{3}{*}{ Australia } & All & 1007 & 30 & 25 & 23 & 23 & 0.01 & 0.13 & 3.4 & -0.3 & -1.6 & -1.6 \\
\hline & Forwards & 526 & 29 & 27 & 23 & 21 & 0.02 & 0.14 & 2.1 & 1.0 & -1.0 & -2.1 \\
\hline & Backs & 481 & 31 & 22 & 22 & 25 & 0.01 & 0.15 & 2.8 & -1.5 & -1.2 & -0.1 \\
\hline \multirow[t]{3}{*}{ England } & All & 1519 & 30 & 25 & 24 & 21 & 0.01 & 0.14 & 4.2 & 0.3 & -1.1 & -3.4 \\
\hline & Forwards & 833 & 29 & 26 & 23 & 21 & 0.01 & 0.12 & 2.4 & 0.7 & -1.1 & -2.0 \\
\hline & Backs & 686 & 32 & 24 & 24 & 20 & 0.01 & 0.18 & 3.6 & -0.4 & -0.4 & -2.8 \\
\hline \multirow[t]{3}{*}{ New Zealand } & All & 1199 & 29 & 26 & 24 & 21 & 0.01 & 0.11 & 2.6 & 0.4 & -0.5 & -2.5 \\
\hline & Forwards & 688 & 29 & 24 & 22 & 24 & 0.06 & 0.10 & & & & \\
\hline & Backs & 511 & 28 & 28 & 27 & 17 & 0.01 & 0.18 & 1.4 & 1.2 & 0.9 & -3.4 \\
\hline \multirow[t]{3}{*}{ South Africa } & All & 2938 & 34 & 26 & 22 & 18 & 0.01 & 0.23 & 9.5 & 0.6 & -2.8 & -7.3 \\
\hline & Forwards & 1598 & 33 & 24 & 23 & 20 & 0.01 & 0.20 & 6.6 & -0.8 & -1.7 & -4.1 \\
\hline & Backs & 1340 & 34 & 27 & 22 & 16 & 0.01 & 0.27 & 6.9 & 1.8 & -2.3 & -6.3 \\
\hline
\end{tabular}

$N$, number of participants; $Q$, quarter of birth; $P, p$-value; $w$, Cohen's w effect size; SR, standardized residual

Table 2. Relative age distribution of rugby union players according to nationality and playing position

\begin{tabular}{|c|c|c|c|c|c|c|c|c|c|c|c|c|}
\hline Country & Population & $\mathbf{N}$ & \% Q1 & $\%$ Q2 & \% Q3 & $\%$ Q4 & $\mathbf{P}$ & $\mathbf{w}$ & SR Q1 & SR Q2 & SR Q3 & SR Q4 \\
\hline \multirow[t]{6}{*}{ Australia } & Front row & 198 & 33 & 23 & 22 & 22 & 0.09 & 0.18 & & & & \\
\hline & Second row & 130 & 23 & 28 & 27 & 22 & 0.65 & 0.11 & & & & \\
\hline & Back row & 198 & 30 & 30 & 21 & 18 & 0.03 & 0.22 & 1.5 & 1.5 & -1.1 & -1.9 \\
\hline & Half backs & 158 & 31 & 21 & 23 & 25 & 0.32 & 0.15 & & & & \\
\hline & Centres & 124 & 34 & 24 & 21 & 21 & 0.14 & 0.21 & & & & \\
\hline & Outside backs & 199 & 30 & 21 & 22 & 27 & 0.20 & 0.15 & & & & \\
\hline \multirow[t]{6}{*}{ England } & Front row & 364 & 26 & 26 & 26 & 22 & 0.61 & 0.07 & & & & \\
\hline & Second row & 187 & 32 & 28 & 20 & 20 & 0.05 & 0.20 & 1.8 & 0.9 & -1.4 & -1.3 \\
\hline & Back row & 282 & 31 & 25 & 22 & 22 & 0.08 & 0.15 & & & & \\
\hline & Half backs & 213 & 30 & 23 & 29 & 18 & 0.05 & 0.19 & 1.5 & -0.7 & 1.2 & -2.0 \\
\hline & Centres & 189 & 30 & 23 & 27 & 20 & 0.21 & 0.15 & & & & \\
\hline & Outside backs & 284 & 35 & 26 & 19 & 20 & 0.01 & 0.25 & 3.2 & 0.5 & -2.1 & -1.5 \\
\hline \multirow[t]{6}{*}{ New Zealand } & Front row & 272 & 30 & 23 & 21 & 26 & 0.17 & 0.14 & & & & \\
\hline & Second row & 161 & 27 & 27 & 21 & 25 & 0.60 & 0.11 & & & & \\
\hline & Back row & 255 & 30 & 24 & 25 & 21 & 0.22 & 0.13 & & & & \\
\hline & Half backs & 179 & 32 & 23 & 23 & 21 & 0.13 & 0.18 & & & & \\
\hline & Centres & 150 & 21 & 29 & 32 & 18 & 0.04 & 0.23 & -1.1 & 1.1 & 1.7 & -1.7 \\
\hline & Outside backs & 182 & 30 & 30 & 26 & 14 & 0.01 & 0.27 & 1.3 & 1.4 & 0.4 & -3.0 \\
\hline \multirow[t]{6}{*}{ South Africa } & Front row & 668 & 33 & 25 & 24 & 19 & 0.01 & 0.20 & 4.0 & -0.2 & -0.8 & -3.0 \\
\hline & Second row & 342 & 35 & 21 & 23 & 20 & 0.01 & 0.24 & 3.7 & -1.5 & -0.6 & -1.7 \\
\hline & Back row & 588 & 33 & 25 & 22 & 20 & 0.01 & 0.19 & 3.8 & 0.0 & -1.5 & -2.3 \\
\hline & Half backs & 480 & 36 & 27 & 23 & 14 & 0.01 & 0.31 & 4.8 & 0.8 & -0.7 & -4.8 \\
\hline & Centres & 352 & 34 & 30 & 20 & 16 & 0.01 & 0.29 & 3.4 & 1.8 & -1.7 & -3.5 \\
\hline & Outside backs & 508 & 33 & 26 & 21 & 19 & 0.01 & 0.22 & 3.7 & 0.5 & -1.6 & -2.7 \\
\hline
\end{tabular}

$N$, number of participants; $Q$, quarter of birth; $P, p$-value; $w$, Cohen's w effect size; SR, standardized residual

front row or second row players was not supported. While the Australian sample showed a RAE in back row players $\left(\chi^{2}(3\right.$, $\mathrm{N}=198)=9.3, \mathrm{p}=0.03, \mathrm{w}=0.22)$ but not in front row or second row players, the English and New Zealand samples showed no RAE for any of the forward units. The South African sample showed a RAE in all three positions (front row, $\chi^{2}(3, \mathrm{~N}=$ ${ }_{668)}=26.0, \mathrm{p}<0.001, \mathrm{w}=0.20$; second row, $\chi^{2}(3, \mathrm{~N}=342)=19.2, \mathrm{p}<$ $0.001, \mathrm{w}=0.24$; back row, $\left.\chi^{2}(3, \mathrm{~N}=588)=21.9, \mathrm{p}<0.001, \mathrm{w}=0.19\right)$. In terms of backline players, RAEs were observed for outside backs in the New Zealand $\left(\chi^{2}(3, \mathrm{~N}=182)=12.9, \mathrm{p}<0.001, \mathrm{w}=0.27\right)$ and English $\left(\chi^{2}(3, \mathrm{~N}=284)=17.4, \mathrm{p}<0.001, \mathrm{w}=0.25\right)$ samples, and for English half backs $\left(\chi^{2}(3, \mathrm{~N}=213)=7.9, \mathrm{p}=0.05, \mathrm{w}=0.19\right)$. Again, the South African sample was distinctive in that an over-representation of players born in the first quarter of the year, and an under-representation of players born in the last quarter of the year was observed for all backline positions (half backs, $\chi^{2}(3, \mathrm{~N}=480)=47.1, \mathrm{p}<0.001, \mathrm{w}=0.31$; centres, $\chi^{2}(3, \mathrm{~N}=352)=$ $30.2, \mathrm{p}<0.001, \mathrm{w}=0.29$; and outside backs, $\chi^{2}(3, \mathrm{~N}=508)=23.8, \mathrm{p}<$ $0.001, \mathrm{w}=0.22)$.

\section{Discussion}

The hypothesis that back row players would be more likely to demonstrate RAEs than front row or second row units was not supported. While the Australian data is equivalent to that reported for French players in a previous study[6], data from the remaining countries showed different patterns. Within the English and New Zealand samples, no forward position showed a pronounced bias, while outside backs showed the strongest RAE. Within the South African sample, all positions showed small to moderate RAEs. It therefore appears that 

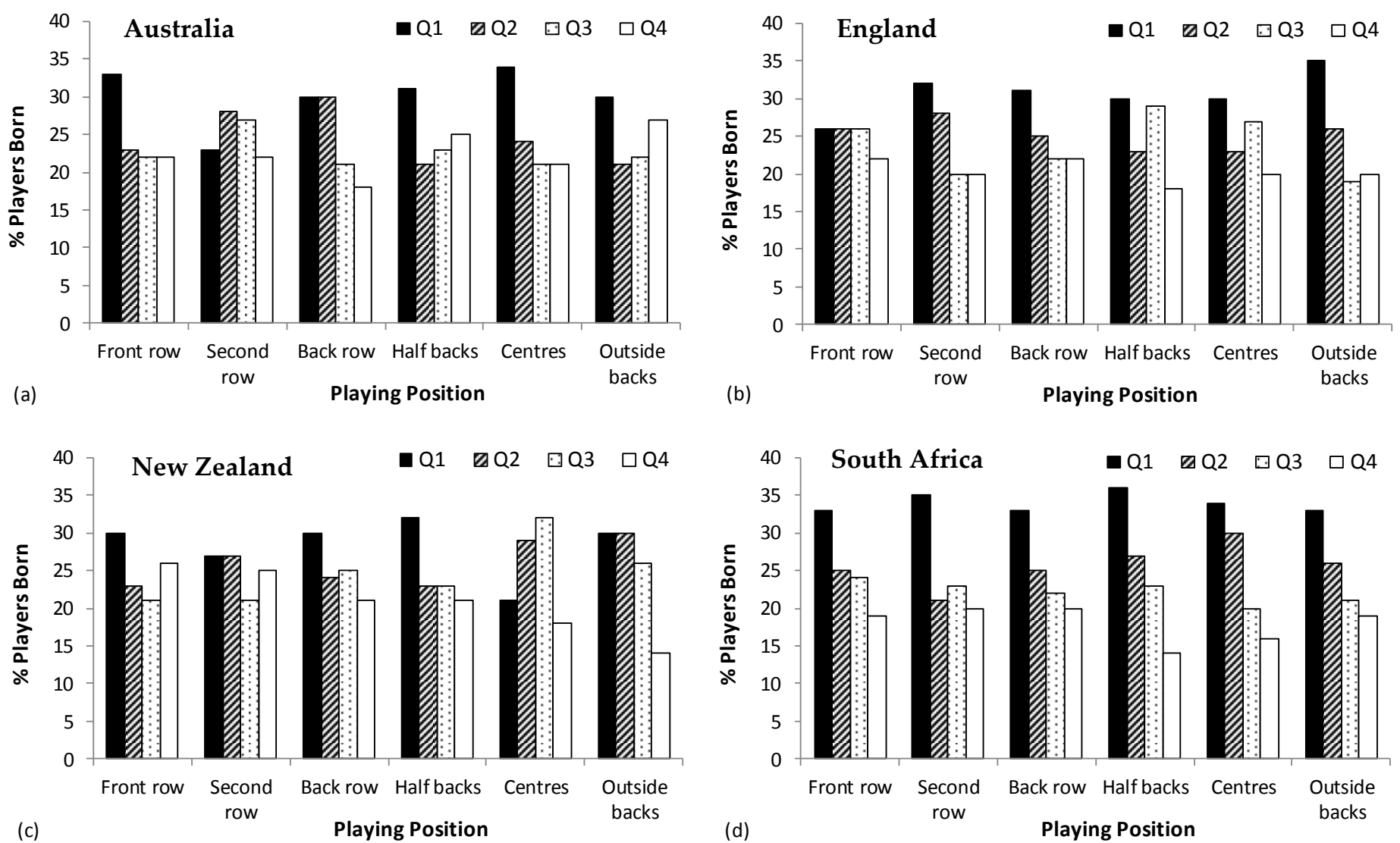

Fig. 1. The influence of playing position on relative age effects in (a) Australian, (b) English, (c) New Zealand, and (d) South African professional rugby players. $Q$, quarter of the year in which players were born.

youth sport culture is potentially more important than playing position for determining who is at risk of RAEs in rugby union. ${ }^{[8]}$

The presence of RAEs at senior level is symptomatic of underlying problems with talent development and youth sport programmes.[1] Specifically, the over-representation of players born in the first quarter of the year and the underrepresentation of players born in the final quarter of the year suggests an inherent inefficiency within talent development systems, whereby players are accessing support and opportunities on the basis of current maturation status rather than future potential. As such, the data tentatively suggest that the talent development processes operating historically within South African rugby were significantly biased towards early maturing players to a greater extent than the talent development processes operating in other countries. Identifying whether progress has been made on this issue would require the analysis of age-graded data. ${ }^{[3]}$

Within rugby union in England, a reversal of the RAE has been identified [9], whereby relatively late-born players were more likely to progress to elite player squad status, despite the existence of a pronounced RAE within the academy cohorts from which these elite players graduated. McCarthy et al. ${ }^{[9]}$ argue that the small minority of players who managed to overcome the disadvantages of being born relatively late in the year benefitted from their additional struggles, likely through the development of a range of psychological skills/characteristics. Care is needed when interpreting this finding, which illustrates the complexity of RAEs. Many youth players may be disadvantaged by the existence of RAEs, including late-born individuals early in their development and early-born individuals later in their development. Given the inevitability of variation in development among youth cohorts, attempts to fully remove RAEs may prove fruitless and even counterproductive. A more appropriate route for talent development programmes may be to focus on developing a broader understanding of the processes influencing both early and late developing players amongst administrators, coaches and the players themselves.

\section{Conclusion}

In summary, the results suggest that RAEs may have influenced talent development in South African rugby to a greater extent than in other major rugby playing nations. Further research is required to determine if RAEs are still present within the South African system, and, if present, the extent to which RAEs influence the process of talent development.

Acknowledgements: Portions of this research were completed while the author was a lecturer at the Institute of Sport, University of Chichester. The author would like to thank Dr PJ Smyth for his helpful comments on an earlier draft of this manuscript.

\section{Conflict of interest: None}




\section{References}

1. Cobley S, Baker J, Wattie N, et al. Annual age-grouping and athlete development: a meta-analytical review of relative age effects in sport. Sports Med 2009;39:235-256. [doi: 10.2165/00007256-200939030-00005]

2. Nutton RW, Hamilton DF, Hutichson JD, et al. Variation in physical development in schoolboy rugby players: can maturity testing reduce mismatch? BMJ Open 2012; 2:e001149. [doi: 10.1136/bmjopen-2012-001149]

3. Lewis J, Morgan K, Cooper S-M. Relative age effects in Welsh age grade rugby union. Int J Sports Sci Coach 2015; 10:797813. [doi: 10.1260/1747-9541.10.5.797]

4. Douglas M. World Rugby: Putting players first. Relative Age Effect. Dublin: World Rugby. n.d. http://playerwelfare. worldrugby.org/?documentid=110 (accessed 29 November 2017).

5. Lambert M, Forbes J, Brown J. Age-group vs weight-group rugby. Cape Town: BokSmart, 2010. http://www.sarugby.co.za/boksmart/pdf/BokSmart\%202010Age\%20vs\%20Weight\%20Category\%20Rugby.pdf
6. Kearney PE. Playing position influences the relative age effect in senior rugby union. Sci Sport 2017; 32:114-116. [doi: 10.1016/j.scispo.2016.06.009]

7. Delahunt E, Byrne RB, Doolin RK, et al. Anthropometric profile and body composition of Irish adolescent rugby union players aged 16-18. J Strength Cond Res. 2013;27: 3252-3258. [doi: 10.1519/JSC.0b013e3182915ea6]

8. Lidor R, Arnon M, Maayan Z, Gershon T, Côté J,. Relative age and birthplace effect in Division 1 female ballgame players the relevance of sport-specific factors. International Journal of Sport and Exercise Psychology 2014;12: 19-33. [doi: 10.1080/1612197X.2012.756232]

9. McCarthy N, Collins D, Court D. Start hard, finish better: further evidence for the reversal of the RAE advantage. J Sport Sci 2016;34:1461-1465. [doi: 10.1080/02640414.2015.1119297] 